\title{
Design of Photovoltaic Power Generation Control System for Practical Training
}

\author{
BaoWen Cao, Lian zhang
}

Department of New Energy Engineering, Tianjin Sino-German University of Applied Sciences, YaShen Road NO.2, HaiHe Education Park, Tianjin, China

caobaowennk@126.com

Keywords: photovoltaic, power generation system, control, training

\begin{abstract}
For the current vocational education training equipment about photovoltaic generation, the control system is lacking of output voltage regulation module. To solve this weakness, this paper puts forward an optimized control system that can provide a constant DC $48 \mathrm{~V}$ voltage source to ensure the stable operation of the whole photovoltaic power generation system.
\end{abstract}

\section{Introduction}

Photovoltaic power generation technology becomes more and more popular in recent years. This technology is one of mean methods that can solve parts of energy reduction problems. Therefore, solar energy plays an important role in Chinese energy structure. Under the lead of Chinese Education Ministry, lots of vocational colleges open relative courses to enhance the skills about photovoltaic generation. The recent researches about photovoltaic power generation control system are mainly focus on the system design ${ }^{[1-2]}$, power quality control ${ }^{[3]}$, control system simulation ${ }^{[4]}$ and maximum power point tracking ${ }^{[5]}$. For the vocational education, most control system of photovoltaic generation is lacking of the output voltage regulation module. To solve this problem, an optimizing control system is designed and explained in this paper.

\section{Design Theories}

\subsection{Control System Composition}

The designed control system contains photovoltaic module, touch screen, I/O controller, DC solid-state voltage regulator, contactors, invertor, battery, watt hour meter, AC/DC voltage sensor, AC/DC current sensor, breaker, fuse, signal light and so no. The DC solid-state voltage regulator makes the output voltage stable in $48 \mathrm{~V}$, and supplies this stable voltage to battery and invertor. The application of this voltage regulator ensures the steady operation and reduces the operation cost of the photovoltaic system.

The electrical drawing of this system is shown as below: 

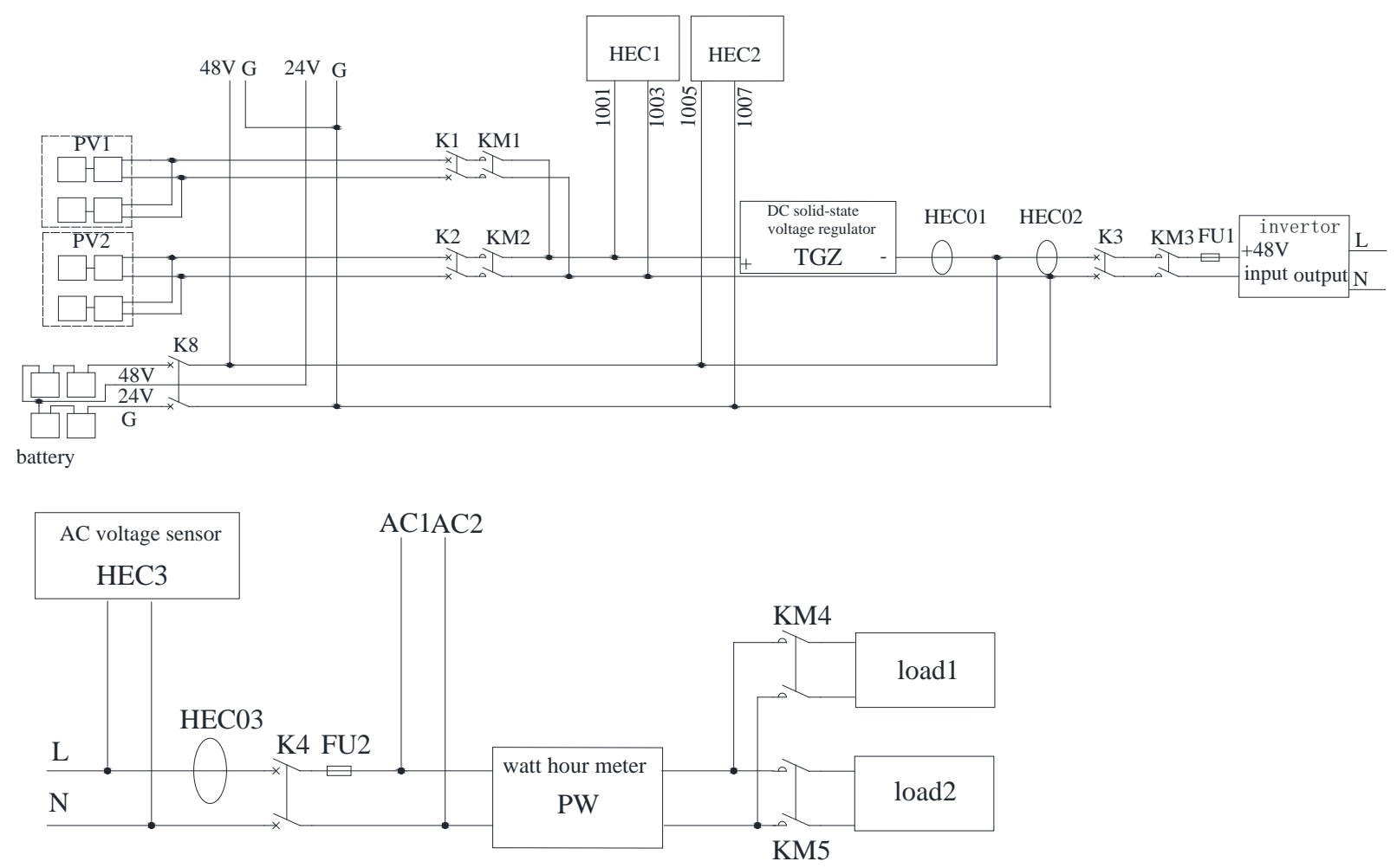

Figure 1. Electrical drawing of control system

\subsection{Basic Design Theories}

(1) Two photovoltaic modules connected in series to be one input, and two inputs connected in parallel in the system. Solid-state voltage regulator converts the two inputs into DC 48V to power the battery and invertor. And invertor changes the two inputs into AC $220 \mathrm{~V}$ to power system load at the same time.

(2) This control system has two I/O controllers. One is to collect voltage and current data of power line and make out analog signal to control DC solid-state voltage regulator. The I/O controller 1 can communicate with touch screen and watt hour meter though RS485 Bus and display the collected data on the screen. The commands on the screen can trigger roils of contactors through DO pin, and then the contactors will be controlled.

(3) The second controller converts $24 \mathrm{~V}$ into $\pm 12 \mathrm{~V}$ and converts analog signals from controller 1 into $0-10 \mathrm{~V}$ voltage signal to control solid voltage regulator.

(4) DC power resources are $48 \mathrm{v}$ and $24 \mathrm{~V}$, and an AC $220 \mathrm{~V}$ source powers system loads and AC contactors. 


\subsection{Control System Operation Theories}

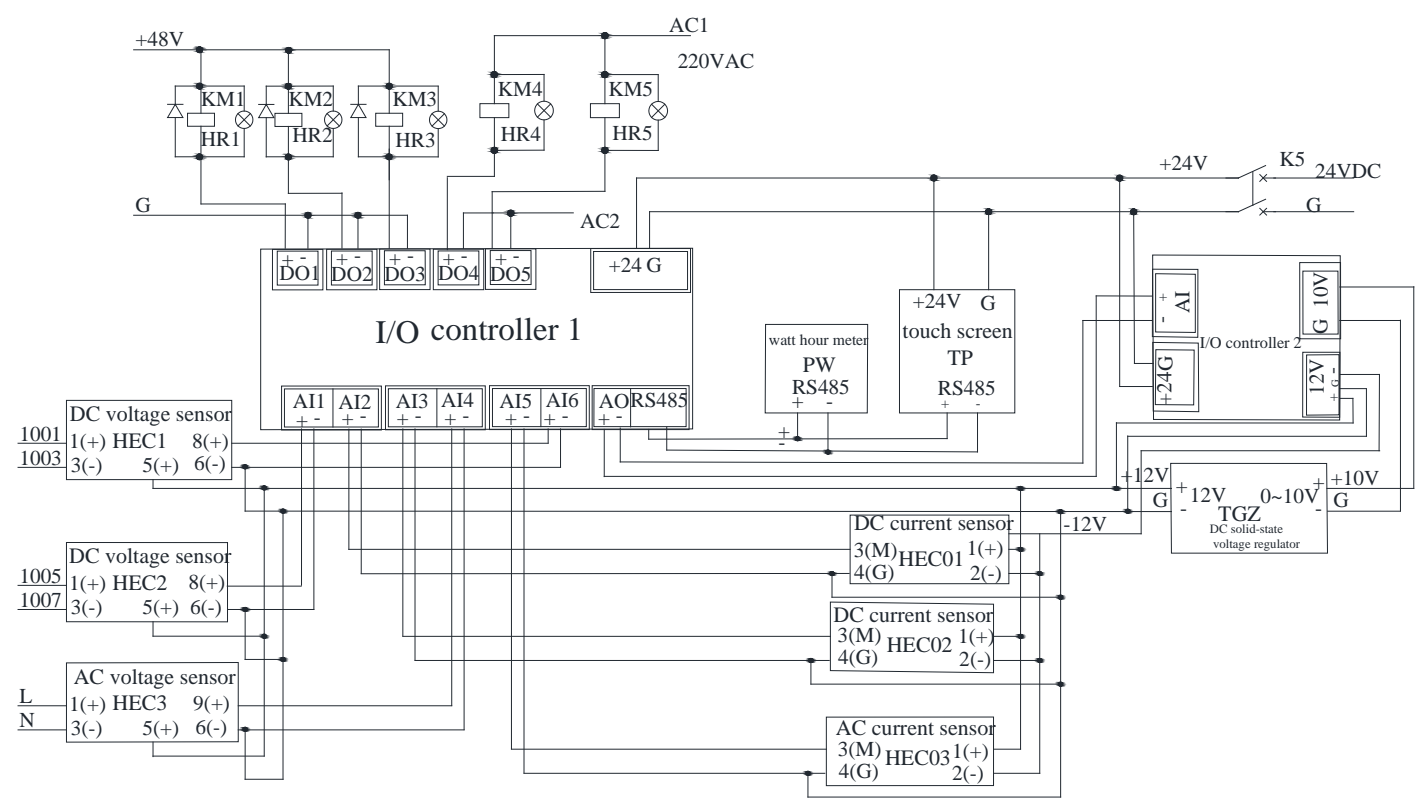

Figure 2. The electrical control drawing of the controller

Figure 2 indicates the specific electrical relations between all system components. From this figure, PV1 and PV2 are the two inputs of this system, and the two inputs go through K1、KM1 and K2、KM2 into DC solid-state voltage regulator TGZ. (K1, K2 are the breakers, KM1, KM2 are the DC contactors.) DC solid voltage regulator outputs a stable DC48V to charge the battery and power the invertor. The charging of battery will be manually controlled by circuit breaker K8. DC breaker K3 and DC contactor KM3 controlled the on/off states of voltage regulator and invertor.The invertor converts DC 48V to AC 220V, breaker K4 and AC contactor KM4, KM5 control load operation.

DC voltage sensor HEC1 and HEC2 monitors the voltage of photovoltaic modules and battery respectively, and AC voltage sensor HEC3 monitors output voltage of invertor. HEC01 and HEC02 are the AC current sensors to monitor output current of solid power regulation module and input current of invertor, current sensor HEC03 is in charge of invertor output voltage monitoring. Fuses FU1 and FU2 are connected in series at the input points of invertor and loads to prevent over current. Watt-hour meter takes charge of load current and voltage monitoring. The battery can provide DC $48 \mathrm{~V}$ and $24 \mathrm{~V}$ to power the DC contactor, touch screen and I/O controller.

I/O controller 1 collects real-time circuit voltage and current from voltage sensor HEC1-3 and current sensor HEC01-03. Then based on the photovoltaic module output voltage, this controller calculates relative analog signal and converts this signal into voltage signal (0-10V) to control solid voltage regulator through $\mathrm{I} / \mathrm{O}$ controller 2 . The other function of $\mathrm{I} / \mathrm{O}$ controller 2 is converting DC24Vto $\pm 12 \mathrm{~V}$ to power voltage sensor HEC1-3 and current sensor HEC01-03.

I/O controller 1, watt-hour meter PW and touch screen TP communicate with each other through RS485 BUS and real-time collected data will be displayed on the touch screen. At the same time, the input commands on touch screen will trigger contactors KM1-KM5 to control the on/off states through DO terminal. Signal lights HR1-5 show the on/off state of relative contactors. The operation of the whole control system will be controlled by a DC breaker K5 which is powered by DC24V.

\section{Conclusion}

This designed control system contains a DC solid-state voltage regulator that makes the whole system operate in a stable $48 \mathrm{~V}$. Then the battery and invertor will not be damaged by the voltage fluctuation and the operation cost will also be reduced. This control system makes the training 
process more effective. It shows the control process of photovoltaic power generation in a simple way, which is a big promotion in the practical training for both teachers and students ${ }^{[6-7]}$. This control system can work on several main experiments, such as energy converting experiment of single crystal photovoltaic, photovoltaic off-network power generation experiment, solar cell energy storage experiment, control system study of photovoltaic experiment, off-network inversion experiment, photovoltaic power generation system study and relative operational experiments.

\section{Acknowledgements}

This research was supported by the Tianjin Education Science "The 13th Five-year" Program Project "Research on the cultivation of systematic talents of the energy specialty" (Project No. VEYP5040) and the Science and Technology Program Project of Jin Nan District Tianjin.

\section{References}

[1] Y Li,Z Yao,J Zhou,K Liu,B Pi,Control System of Small-scale Photovoltaic Power Generation,Microcontrollers \& Embedded Systems,2015

[2] Dong-Hui Li, Yi-Lin Wang,Da-jun Zhou,Gongnan Xie,Research on a New Control Scheme of Photovoltaic Grid Power Generation System,Journal of Applied Mathematics,2014

[3] Wei Wei Song,Hybrid System for Photovoltaic Power Generation and Power Quality Control,Applied Mechanics and Materials,Vol. 734,759-770,2015

[4] Yong Tao Dai,You Hui Xie,Wang Kun Li, Research on Control Simulation of Photovoltaic Power Generation System Based on MPPT,Advanced Materials Research,Vols. 724-725, 74-77, 2013

[5] Yi Zong Dai,De Jun Miao,A Research on the Fuzzy Control with Genetic Algorithm for Maximum Power Point Tracking of Photovoltaic Power System,Applied Mechanics and Materials, Vols. 303-306, 1153-1157, 2013

[6] Zhong Nian Li, Yun Feng Yue, Guo Liang Chen,Construction \& Operation for the Training Base about Application Technology on New Energy Resuorce,Advanced Materials Research,Vols. 524-527, 2895-2898, 2012

[7] SUN Chuanqing,The Development and Application of a Training Base for the Installation and Adjustment of Photovoltaic Power Generation Systems,International Journal of Advanced Culture Technology, Vol.4,37-50,2016 